\title{
Branching with Local Probability as a Paradigm of Self-Organized Criticality
}

\author{
Stefan Hergarten* \\ Institut für Angewandte Geowissenschaften, TU Graz, A-8010 Graz, Austria \\ Institut für Erdwissenschaften, Karl-Franzens-Universität Graz, A-8010 Graz, Austria
}

(Received 8 May 2012; published 4 October 2012)

\begin{abstract}
A self-organized critical branching process based on a local interaction rule is presented. In accordance with the self-organized branching process model introduced by Zapperi, Lauritsen, and Stanley, its eventsize distribution follows a power law with scaling exponent $\tau=\frac{3}{2}$, but the new model does not require a global variable to self-organize to a critical point. The self-organized critical behavior of the model seems to be extremely robust. The model may be seen as a new paradigm for progressive mechanical failure (e.g., earthquakes or landslides) or other avalanching phenomena, and perhaps even for self-organized criticality in general.
\end{abstract}

DOI: 10.1103/PhysRevLett.109.148001

PACS numbers: $45.70 . \mathrm{Ht}, 05.65 .+\mathrm{b}, 05.70 . J \mathrm{~J}$

Introduction.-The self-organized branching process (SOBP) model [1] has perhaps been the most successful approach to bridge the gap between self-organized criticality (SOC) and branching processes. It was originally designed as a mean-field approach to understand the dynamics of the Bak-Tang-Wiesenfeld (BTW) model [2,3] (strictly speaking, Manna's two-state version of the model [4]), so that it can be viewed as some kind of a model for a model.

The propagation of avalanches in the SOBP model follows a simple binary branching process [5]. An avalanche starts with one active site that relaxes with a given probability $p$. If the site relaxes, it triggers two other sites, and each of them relaxes with the same probability $p$. Those of the two sites which relax trigger two further sites each, and so on. Avalanche sizes $s$ are measured in terms of the number of relaxed sites. This part of the model has a (tuned) critical point at $p=p_{c}=\frac{1}{2}$ [5], where the probability mass function of the event-size distribution follows a power law,

$$
f(s) \propto s^{-\tau},
$$

with a scaling exponent $\tau=\frac{3}{2}$.

As a new idea compared to previous approaches in this direction [6-8], the probability $p$ is not a given tuning parameter in the SOBP model, but a global variable. The evolution of this variable through time is motivated from the behavior of the BTW model. In the BTW model, the mean value of the local variable (number of grains) increases slowly through time, and decreases only when the avalanches reach the boundary. In analogy to this, the

Published by the American Physical Society under the terms of the Creative Commons Attribution 3.0 License. Further distribution of this work must maintain attribution to the author(s) and the published article's title, journal citation, and DOI. authors of the SOBP model derived a model rule where the global variable $p$ increases in general, but decreases as a consequence of a large avalanche.

It was found that the SOBC model self-organizes towards a quasisteady state that fluctuates around the critical point $p=p_{c}=\frac{1}{2}$ of the binary branching process. Size distribution of the avalanches follows the same distribution as the branching process at its critical point. In this sense, the SOBP model is self-organized critical.

However, the presence of a global variable may be seen as a weakness of the model. With respect to the original focus, a mean-field model for the BTW model, this shall not be a serious problem. But if we consider the SOBP model as a model in itself or as a fundamental type of SOC in branching processes, it becomes difficult to interpret the rule for the evolution of the global variable physically. Viewed under the aspects of SOC, the global variable introduces an infinite (or system-wide) correlation length a priori, so that the SOBP model, strictly speaking, does not organize itself towards a state with infinite correlation length.

Some extensions of the SOBP model towards systems without conservation have been proposed [9-11], either with regard to real-world phenomena such as neuronal avalanches, or in order to obtain a mean-field model for (apparently) self-organized critical systems without conservation. The nonconservative version of the OlamiFeder-Christensen (OFC) earthquake model [12] is perhaps the most prominent example of such a system, although it was found that self-organization towards a critical state is much more complicated here [13,14]. Several other applications of critical branching processes have been published, particularly in astrophysics $[15,16]$ and life sciences $[17,18]$. However, these models involve the probability $p$ directly as a tuning parameter, assume a suitable statistical distribution of $p$, or require a suitable averaging over different values of $p$, and are thus tuned rather than self-organized critical. In summary, there still seems to be a gap between branching processes and SOC. 
On one side, there are some models based on physically reasonable rules, but require some degree of tuning to become critical, and on the other side there is the SOBP model that can be related to real-world phenomena only under very specific physical conditions.

Branching with local, preserved probability.-The approach presented in the following takes a rather simple step ahead by replacing the global probability $p$ with a local probability $p_{i}$ assigned to each site $i$. If a site is successfully triggered, it relaxes conservatively towards two other sites: half of its probability $p_{i}$ is transferred to each of these sites, while its own probability is set to zero. Then, these two sites are triggered, too. If a site is unsuccessfully triggered, it simply keeps its probability, of course, including the probability received before it was triggered. Other branching numbers $n_{b}>2$ can be considered, too, where conservation implies that an amount $\frac{p_{i}}{n_{b}}$ is transferred to each of the triggered sites. For $n_{b}=4$, the relaxation rule is exactly the same as in the conservative case of the OFC model and very similar to that of the BTW model, while for case $n_{b}=2$, it is similar to the relaxation in Manna's [4] version.

The model requires a rule for initial triggering of avalanches, and, as it is conservative, a loss at hypothetic boundaries. Initial triggering is realized simply by selecting a site randomly, setting its value $p_{i}$ to a given value $p_{0}$, and forcing this site to relax. Instead of forcing the site to relax, we could also assume that this site relaxes at its probability $p_{i}=p_{0}$, but this makes no significant difference. Alternatively, a given increment $\delta p$ could be applied to a randomly selected site, and then let this site relax, depending on its new probability $p_{i}$. Concerning the hypothetic boundary, we could follow the SOBP model and restrict the duration of avalanches, i.e., the total height of the branching tree. But as the new model requires a given total number of sites, this would introduce a second cutoff scale. Let us therefore assume that a site can participate in an avalanche only once. If a site is triggered for the second time during an avalanche, is is simply unloaded $\left(p_{i}=0\right)$ without triggering further sites.

In this Letter, the random-neighbor version of the model is examined. This means that each relaxing site randomly selects two children to be triggered. Strictly speaking, even the random-neighbor version is non-Abelian, but the rule that a site cannot take part in an event twice helps to reduce the potential influence of the order of relaxations. The results shown in the next section were computed using breadth-first order, but depth-first order yields the same results within the statistical variation.

Results.-Figure 1 shows the probability mass function of the avalanche sizes for $p_{0}=\frac{1}{2}$ and different system sizes from $n=2^{15}=32768$ to $n=2^{30} \approx 10^{9}$. The probabilities were estimated by logarithmic binning of $2^{30}$ events with 100 bins per decade after a quasisteady state was reached.

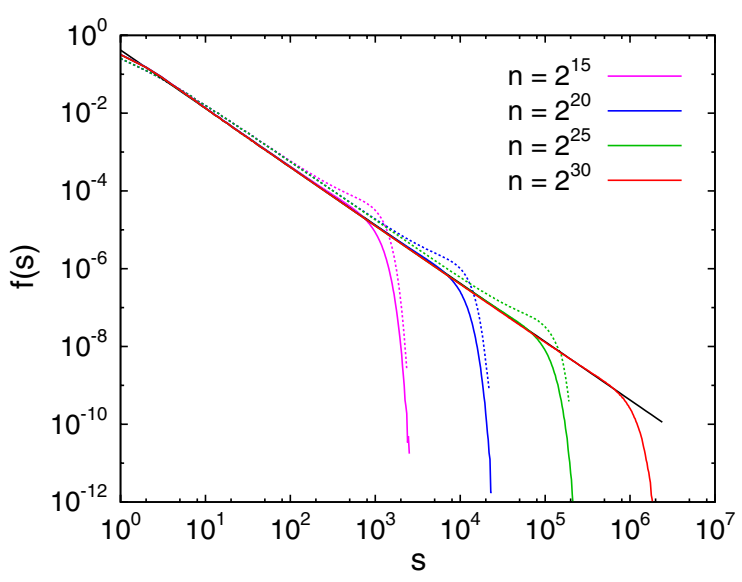

FIG. 1 (color online). Probability mass function of the eventsize distribution for $p_{0}=\frac{1}{2}$ and different system sizes. The black line corresponds to a power law with a scaling exponent $\tau=\frac{3}{2}$. The dashed lines illustrate the distributions resulting from a critical branching process $\left(p=\frac{1}{2}\right)$ at the same system size (only for $n \leq 2^{25}$ ).

Similarly to the SOBP model, the new model selforganizes to a state where the event-size distribution approaches a power law with a scaling exponent $\tau=\frac{3}{2}$ in the limit of infinite system size. A moment analysis results in $\tau=1.499(15)$ and a finite-size scaling with $n^{0.662(10)}$, which is presumably $n^{2 / 3}$, and thus a scaling of the mean event size like $n^{1 / 3}$. Mean-field approximations of sandpile models [19] yield $\tau=\frac{3}{2}$, a finite-size scaling with $L^{4}$, and a scaling of the mean event size like $L^{2}$, where $L$ is the length scale of the system. Thus, the scaling behavior of the new model coincides with the mean-field sandpile approximation at a spatial dimension of six.

As Fig. 2 illustrates, the self-organization of the model is not contingent on the choice of $p_{0}=p_{c}=\frac{1}{2}$. The choice of $p_{0}$ mainly affects the behavior at small event sizes. For small values of $p_{0}$, the avalanches will most probably get

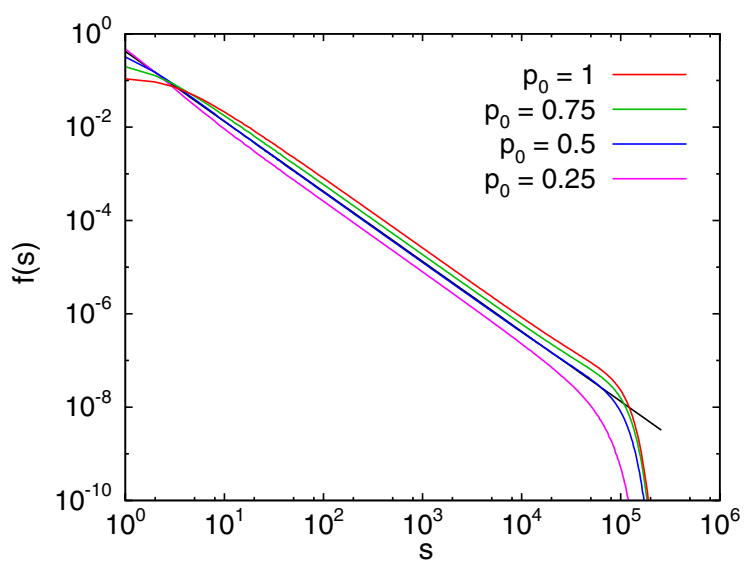

FIG. 2 (color online). Probability mass function of the eventsize distribution for different values of $p_{0}\left(n=2^{25}\right)$. The black line corresponds to a power law with $\tau=\frac{3}{2}$. 


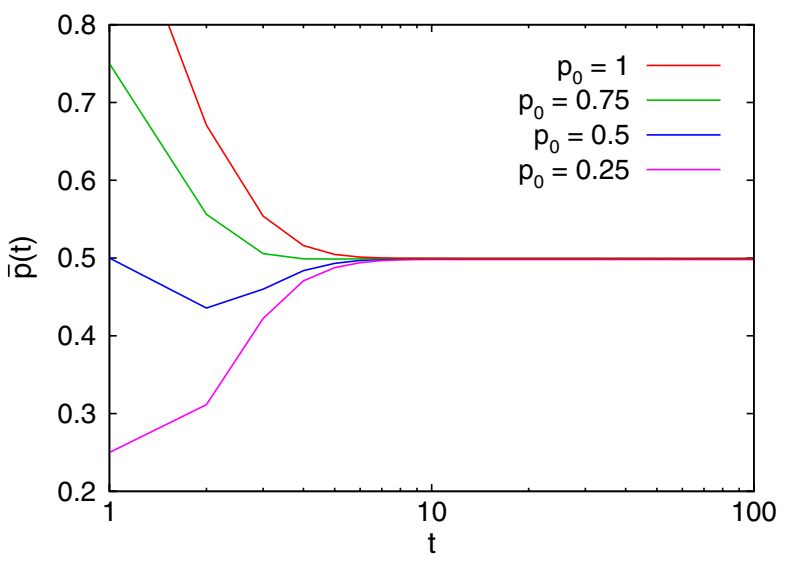

FIG. 3 (color online). Mean probabilities $\bar{p}(t)$ during the avalanches, where $t$ is the generation number within the avalanche.

stuck quite at the beginning, leading to a higher number of small events, and vice versa. However, the power-law scaling itself is unaffected by the choice of $p_{0}$, at least for the range $\frac{1}{4} \leq p_{0} \leq 1$ investigated here. Figure 3 shows that the mean value of $p_{i}$ rapidly approaches a value close to $\frac{1}{2}$ during an event. Here, time within the event is simply the generation number of triggered sites (i.e., the depth in the avalanche tree plus one), so that the initial site corresponds to $t=1$, its children have $t=2$, and so on. In this sense, $\bar{p}(t)$ is the mean probability of all the sites triggered in generation $t$ after having received their increment in $p_{i}$, but regardless of the question whether they relax or not. When computing this mean probability, it must be taken into account that $p_{i}$ is the probability only if $p_{i} \leq 1$, and the probability is one for $p_{i}>1$.

Averaging over all sites with $t>20$ (in order to avoid the effects of $p_{0}$ ) yields the overall mean probabilities $\bar{p}=$ 0.4922 for $n=2^{15}, \bar{p}=0.4974$ for $n=2^{20}, \bar{p}=0.4992$ for $n=2^{25}$, and $\bar{p}=0.4997$ for $n=2^{30}$. These results suggest that the branching process is subcritical for finite system sizes, but approaches the critical point in the limit $n \rightarrow \infty$. The mean probability approaches the critical value $p_{c}=\frac{1}{2}$ inversely proportionally to the mean event size, namely, $p_{c}-\bar{p} \sim n^{-(1 / 3)}$ for $n \rightarrow \infty$. As illustrated in Fig. 1, the subcriticality at finite system sizes helps to maintain the power-law distribution. In case of a tuned critical branching process at the same system size (dashed lines), with the same rules for the loss at the hypothetic boundaries (implemented by artificially keeping all probabilities at $p_{i}=p_{c}=\frac{1}{2}$ ), a strong bump in the distribution occurs at the finite-size cutoff, resulting from large avalanches being cut by finite-size effects. Apart from this, the event-size distribution approaches a power law at small event sizes much faster in the model with variable $p_{i}$ than for the tuned critical branching process. This may arise from the self-organization of the values $\bar{p}(t)$ at small $t$ (Fig. 3). In sum, it seems that the distribution of the probabilities $p_{i}$ not only self-organizes to generate a

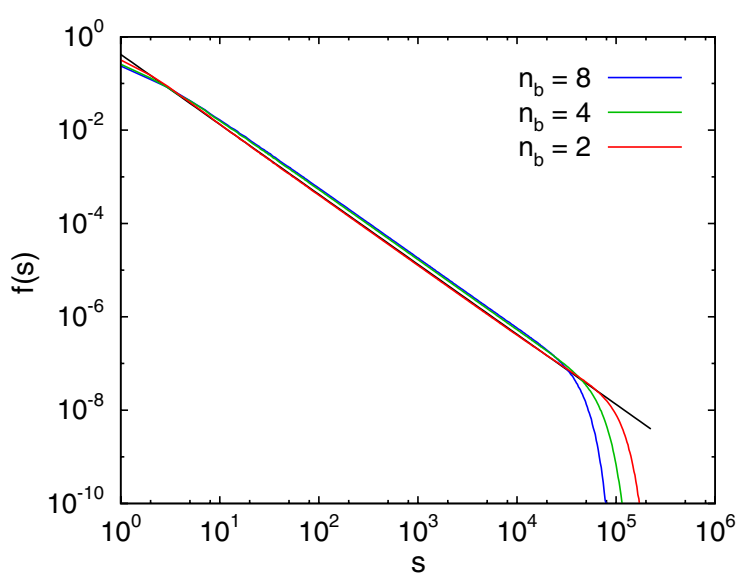

FIG. 4 (color online). Probability mass function of the eventsize distribution for different branching numbers $n_{b}\left(p_{0}=\frac{1}{2}\right.$, $n=2^{25}$ ). The black line corresponds to a power law with $\tau=\frac{3}{2}$.

power-law event-size distribution in the limit $n \rightarrow \infty$, but also maintains a very clear power-law distribution at finite system sizes.

As Fig. 4 illustrates, the branching model is selforganized critical for branching numbers $n_{b}>2$, too, and the scaling exponent $\tau=\frac{3}{2}$ persists. This may be surprising at first, because lattice-based models of SOC show a dependence of $\tau$ on the spatial dimension $d$, and it is straightforward to relate dimension and branching numbers by a relation like $n_{b}=2^{d}$. However, mean-field approximations of these models yield scaling exponents independent of $d$, and the random-neighbor version of the new model is in principle a mean-field model.

In contrast to the universal exponent $\tau$, the statistical distribution of the values $p_{i}$ of the triggered sites must strongly depend on $n_{b}$, as the portions of $p_{i}$ that are transferred before the site is triggered decrease with the increasing branching number. Figure 5 shows the corresponding probability density. The data were derived from $2^{30}$ avalanches with $n=2^{25}$ each, where all triggered sites $i$ with

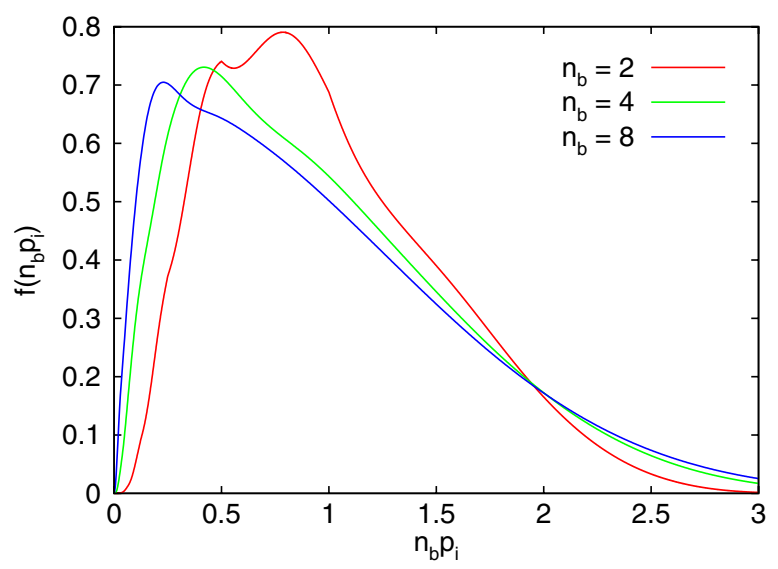

FIG. 5 (color online). Probability density of $n_{b} p_{i}$ for different values of $n_{b}$. 
$t>20$ (as above, in order to avoid the influence of $p_{0}$ ) were evaluated. Instead of the distribution of the values $p_{i}$ itself, the distribution of $n_{b} p_{i}$ was plotted, because it has a mean value of one in the critical state. In case of tuned criticality $\left(p_{i}=\frac{1}{n_{b}}\right)$, this distribution would peak only at $n_{b} p_{i}=1$. The distribution becomes wider as $n_{b}$ increases, which means that both low and high values of $n_{b} p_{i}$ occur more frequently. This means that the randomness in the triggering process is reduced, and once a site is randomly selected to be triggered, it is more or less clear whether it relaxes or not. So the distribution of the values $p_{i}$ is nonuniversal, and moves away from the respective tuned critical branching process if $n_{b}$ increases, but obviously organizes so that the system maintains its self-organized critical behavior with different degrees of randomness in the triggering process.

Preliminary further simulations (not shown here) indicate that the model is robust even against other alterations in its rules. The random-neighbor relations can apparently be replaced by a deterministic graph without changing the results as long as a site cannot receive multiple increments in $p_{i}$ before it is triggered. Even more interesting, it appears that the probability of relaxation need not coincide exactly with the conservative variable $p_{i}$. If, for example, this probability is $p_{i}^{\alpha}$ instead of $p_{i}$, the self-organized critical behavior and the scaling exponent persist, of course, with a different statistical distribution of $p_{i}$. This was tested for $n_{b}=2$ and $n_{b}=8$ and for several values of $\alpha$, including the case of $\alpha=\infty$, corresponding to a steplike function, where the triggering process becomes completely deterministic. This robustness makes the model interesting for a wide range of processes since preserved properties are present in many processes. For instance, total force or stress should be preserved in mechanical failure phenomena. Force or stress affects the probability of failure, but there will be no linear relationship between stress or force and the probability of failure in general. A more thorough investigation of the model's robustness will be the subject of a subsequent study.

Conclusions.-Inspired by the SOBP model, a selforganized critical branching process involving a conservative local variable has been presented. In analogy to the SOBP model and to a branching process tuned to its critical point, this model yields a power-law avalanche-size distribution with a scaling exponent $\tau=\frac{3}{2}$. The model's behavior seems to be extremely robust. From its basic rules, the new model is almost as simple as the most widespread models of SOC, namely, the BTW model [2,3], the OFC model [12], and the Drossel-Schwabl forest-fire model [20]. From its behavior, it seems to be even much more simple than these models. So this new self-organized branching model might even be seen as a new paradigm for the relationship between branching processes and SOC in the context of avalanching, progressive mechanical failure, and perhaps even other phenomena.

This study was funded by the Austrian Science Fund (FWF): P19733-N10 and EUROCORES TopoEurope I152.

*stefan.hergarten@uni-graz.at; http://hergarten.at

[1] S. Zapperi, K. B. Lauritsen, and H. E. Stanley, Phys. Rev. Lett. 75, 4071 (1995).

[2] P. Bak, C. Tang, and K. Wiesenfeld, Phys. Rev. Lett. 59, 381 (1987).

[3] P. Bak, C. Tang, and K. Wiesenfeld, Phys. Rev. A 38, 364 (1988).

[4] S. Manna, Physica (Amsterdam) 179A, 249 (1991).

[5] T. E. Harris, The Theory of Branching Processes (Dover, New York, 1989).

[6] P. Alstrøm, Phys. Rev. A 38, 4905 (1988).

[7] K. Christensen and Z. Olami, Phys. Rev. E 48, 3361 (1993).

[8] R. García-Pelayo, Phys. Rev. E 49, 4903 (1994).

[9] K. B. Lauritsen, S. Zapperi, and H. E. Stanley, Phys. Rev. E 54, 2483 (1996).

[10] D. E. Juanico, C. Monterola, and C. Saloma, Phys. Rev. E 75, 045105 (2007).

[11] D. E. Juanico and C. Monterola, J. Phys. A 40, 9297 (2007).

[12] Z. Olami, H. J. S. Feder, and K. Christensen, Phys. Rev. Lett. 68, 1244 (1992).

[13] A. A. Middleton and C. Tang, Phys. Rev. Lett. 74, 742 (1995).

[14] S. Hergarten and R. Krenn, Nonlinear Proc. Geophys. 18, 635 (2011).

[15] A. L. MacKinnon, K.P. MacPherson, and L. Vlahos, Astron. Astrophys. 310, L9 (1996).

[16] K.P. MacPherson and A.L. MacKinnon, Astron. Astrophys. 350, 1040 (1999).

[17] C. Adami and J. Chu, Phys. Rev. E 66, 011907 (2002).

[18] C. Haldeman and J. M. Beggs, Phys. Rev. Lett. 94, 058101 (2005).

[19] A. Vespignani and S. Zapperi, Phys. Rev. Lett. 78, 4793 (1997).

[20] B. Drossel and F. Schwabl, Phys. Rev. Lett. 69, 1629I (1992). 